\title{
Pedestrian Serviceability Index Including Pedestrians on
} Vehicle-Path

\author{
Mukti Advani ${ }^{1}$, Purnima Parida ${ }^{2}$ and Sobhana Patnaik ${ }^{3}$ \\ 1. Senior Scientist, Transportation Planning, CSIR—Central Road Research Institute, New Delhi 110025, India; \\ 2. Senior Principal Scientist, Transportation Planning, CSIR—Central Road Research Institute, New Delhi 110025, India; \\ 3. Assistant Professor, Civil Engineering Department, C.V.Raman College of Engineering, Bhubneshwar 752054, India
}

\begin{abstract}
In Indian mixed traffic, a common scenario is when motorized vehicles, non-motorized vehicles including pedestrians use the same road space. In such conditions, if PLOS (pedestrian level of service) is measured using conventional methods, i.e., considering pedestrians walking on footpath only; leads to unrealistic results as most of the time pedestrians are seen walking on the path dedicated for vehicles, i.e., vehicle-path. The decision making behavior of pedestrians regarding walking on footpath or on vehicle-path is influenced by the quality of footpath as well as the characteristics of traffic on vehicle-path and the presence of vendors in the street. Hardly any research carried out on measuring pedestrians' serviceability on road considering pedestrians walking on vehicle-path. This study attempts to estimate the effect of various parameters such as intensity of vehicular traffic on the vehicle-path, presence of street vendors on the vehicle-path, etc., on pedestrians' decision of walking on footpath or on vehicle-path based on data collected at two locations in New Delhi, India, by using various statistical methods, especially binary logistic regression. One of the locations is an urban two-lane undivided road with footpath on both sides, a part of National Highway-2, another one is a four-lane divided road with footpath on both sides which is a typical arterial road. It has been found that vendors play a significant role in pedestrians' decision making regarding walking on footpath or on vehicle-path. Study proposes a model to measure pedestrians' serviceability on a road where pedestrians may be walking on the vehicle-path even in the presence of footpath.
\end{abstract}

Key words: Pedestrian, India, serviceability, vehicle-path, foot-path.

\section{Introduction}

Pedestrian facilities are vital components in a community's transportation infrastructure. However, it becomes an issue of major concern when in the presence of such pedestrians' facility, pedestrians prefer to walk on the space allocated for motorized vehicles, i.e., vehicle-path. Design gaps, lack of proper maintenance and cleanliness in the pedestrian facility, are obvious reasons for inducing such behavior. It leads to potential conflicts or safety issues arising among pedestrians, bicyclists and motorists. In such scenarios; pedestrians' level of service measurement becomes complex as most of the methods available for the measurement of pedestrian level of service adhere to

\footnotetext{
Corresponding author: Mukti Advani, senior scientist; research field: transportation planning. E-mail: mukti7@gmail.com.
}

the designated pedestrian facilities only. Such evaluation process would definitely be misleading in making a decision on the pedestrian level of service provided by the facility, when most of the pedestrians are not using the facility allotted to them. Hence, there is a need to study various parameters influencing the pedestrians' decision for walking on the vehicle-path, even in the presence of footpath. Since, situations like this are quite common in urban Indian roads, a methodology for measuring pedestrians' serviceability considering pedestrians walking on vehicle-path as well as on footpath is required to be developed for a more realistic measurement.

\section{Literature Review}

Conventionally, performance measures of pedestrians' sidewalk facilities are based on "walking space". As mentioned by Asadi-Shekari et al. [1], there 
are two common approaches for evaluating PLOS (pedestrian level of service): the first can be defined as a capacity-based model and the second is a roadway characteristic-based model. Capacity based methods are based on the principles of highway capacity which have been suitably adjusted to evaluate pedestrian facilities which are helpful in planning pedestrian facilities but provide little information regarding acceptability by pedestrians. Roadway Characteristics based methods are based on the characteristics of the walkways or pedestrian facilities. These methods are based on pedestrians' perceptions and also attempts to quantify the comfort level of pedestrians while encountering certain roadway characteristics. Fruin [2] suggested a PLOS measurement method based on sidewalk capacity and pedestrian volume. This PLOS model was one of the first attempts to measure pedestrian level of service and was based on the area module, i.e., space available per pedestrian. HCM (Highway Capacity Manual) [3, 4] gives the PLOS model based on Fruin's methodology and the majority of pedestrian facility design guidelines are based on the US Highway Capacity Manual. However, there are many other studies which consider HCM method to be insufficient as it does not consider the qualitative parameters. For example, the impacts of environmental indicators on pedestrian facilities were examined by Lautso and Murole [5] extended this PLOS measurement method by incorporating several important variables into the calculation of PLOS. Sarkar [6] suggested a method to qualitatively measure PLOS based on six factors, i.e., safety, security, convenience and comfort, continuity, system coherence and attractiveness. He proposed six service levels: these levels range from $\mathrm{A}$ to $\mathrm{F}$, and the most pedestrian-friendly street is indicated by PLOS A. However, being a qualitative method, it does not provide ease to measure each parameter. Henson [7] proposed a comprehensive review of PLOS measurements. He listed several prominent factors that have an effect on PLOS (e.g., comfort, convenience, safety, security and economy) based on the previous literature. Gallin [8] developed a model which consists of three main categories that affect PLOS, i.e., physical characteristics (path width, surface quality, obstructions, crossing opportunities), location factors (connectivity, path environment) and user factors (pedestrian volume, mix of path users, security). The model not only evaluates the PLOS of a pedestrian path but also determines the factors that contribute to a low or high LOS (level of service). Mozer [9] suggested a PLOS model based on four primary factors, i.e., walk area width-volume, walk area-outside lane buffer, outside lane traffic volume and outside lane motor vehicle speed. In addition, three secondary factors were considered: walk area penetration, heavy vehicle volume and intersection waiting time. In a Handbook [10], LOS refers to four variables relevant with the pedestrian level of service, i.e., existence of a sidewalk, lateral separation of pedestrians from motorized vehicles, motorized vehicle volumes and motorized vehicle speeds. Landis et. al. [11] quantifies pedestrians' perception of safety and comfort in the roadside environment. This quantification provides a measure of how well roadways accommodate pedestrian travel safely. It includes factors such as lateral separation elements between pedestrian and motor vehicle traffic (presence and width of sidewalk, presence of on-street parking or bike lane, width of outside travel lane), motor vehicle traffic mix, volumes, and speeds. Jensen [12] claims that the variables with the largest effect on pedestrian satisfaction are the type and width of walking area and the distance from motor vehicles in nearest drive lane. In his model, qualitative variables are also included (the type of roadside development the presence of shading trees). Further, Jaskiewicz [13] and Abley and Turner [14] developed various models, including both quantitative and qualitative variables that refer to aesthetics, safety as well as design elements and traffic characteristics. Muraleetharan et al. [15] refer to eight criteria: sidewalk width and separation (buffer area, shoulder or 
bike lane, on-street parking), obstructions, flow rate, number of bicycle passing and opposing events, space at corner, crossing facilities, turning vehicles and delay. Petritsch et al. [16] and Dowling et al. [17] developed models including a long list of variables influencing pedestrians' sense of safety or comfort along the facility. Some of them refer to: proximity to the travel lanes (i.e., separation between the pavement and the sidewalk, width of the sidewalk), perceived conflicts at intersections (with turning vehicles), and perceived threat exposure when crossing roadways or driveways (i.e., crossing distance, presence of crosswalk, other traffic control devices and of median) and delays at intersections.

For all the existing methods, it is to be noted that these are primarily developed for the pedestrians sidewalks excluding or including parameters related to traffic on adjacent motorized lanes. However, on Indian roads, it is not uncommon to see few pedestrians walking on motorized path (vehicle-path) apart from the few walking on footpath on the same stretch of the road. If most commonly used space, flow and speed based LOS method is applied to evaluate such situations (i.e., few pedestrians walking on footpath as well as few on vehicle-path), it may provide better LOS which is contradictory as it considers only those pedestrians who are walking on footpath. Since, pedestrian LOS is operationally defined as freedom to maneuver, it would be logical to conclude that, a pedestrian facility provides a high LOS if few pedestrians are present on footpath. Hence, there is a need to include all pedestrians, i.e., pedestrians walking on footpath as well as on vehicle-path to capture the ground reality in quantification [18]. This study focuses on developing such quantification method which can be used to measure serviceability level of all the pedestrians walking on footpath and/or on vehicle-path.

\section{Study Focus and Scope}

This study focuses on understanding the decision making behavior of pedestrians with respect to the choice they make for walking on footpath or on vehicle-path in the presence of footpath. Study further proceeds to quantify level of serviceability by developing PSI (pedestrians' serviceability index) including pedestrians walking on footpath as well as on vehicle-path. The scope of the present study is limited to sidewalks only.

\section{Research Methodology}

Fig. 1 presents the broad methodology used for present study.

As shown in Fig. 1, study focus was fixed based on ground observations regarding pedestrians' walking on footpath/vehicle-path and available literature regarding methods to measure pedestrians' level of service on these paths. Methodology adopted for this study includes five broad steps. The first step is data collection, i.e., to collect video data for classified traffic volume count on vehicle-path, number of pedestrians walking on footpath and on vehicle-path, quality observations for vehicle-path and footpath and questionnaire design followed by pedestrians' interviews. The second step involves the extraction of sample snapshots (i.e., a photograph taken from video) to cover the widest possible range of space occupied by vehicles at selected locations. This has been used to extract details of space occupied by vehicles, space occupied by street vendors and number of pedestrians walking on footpath and on vehicle-path in Step 3. Step 4 focuses on developing the relationship between space occupied by vehicles and pedestrians' which is further analyzed to understand decision making behavior of pedestrians with respect to space occupancy (by vehicles and street vendors) considering the quality parameters along with pedestrians' stated opinions. Finally in Step 5, a model has been developed to measure the pedestrians' serviceability for all the pedestrians walking on footpath as well as on vehicular path. 


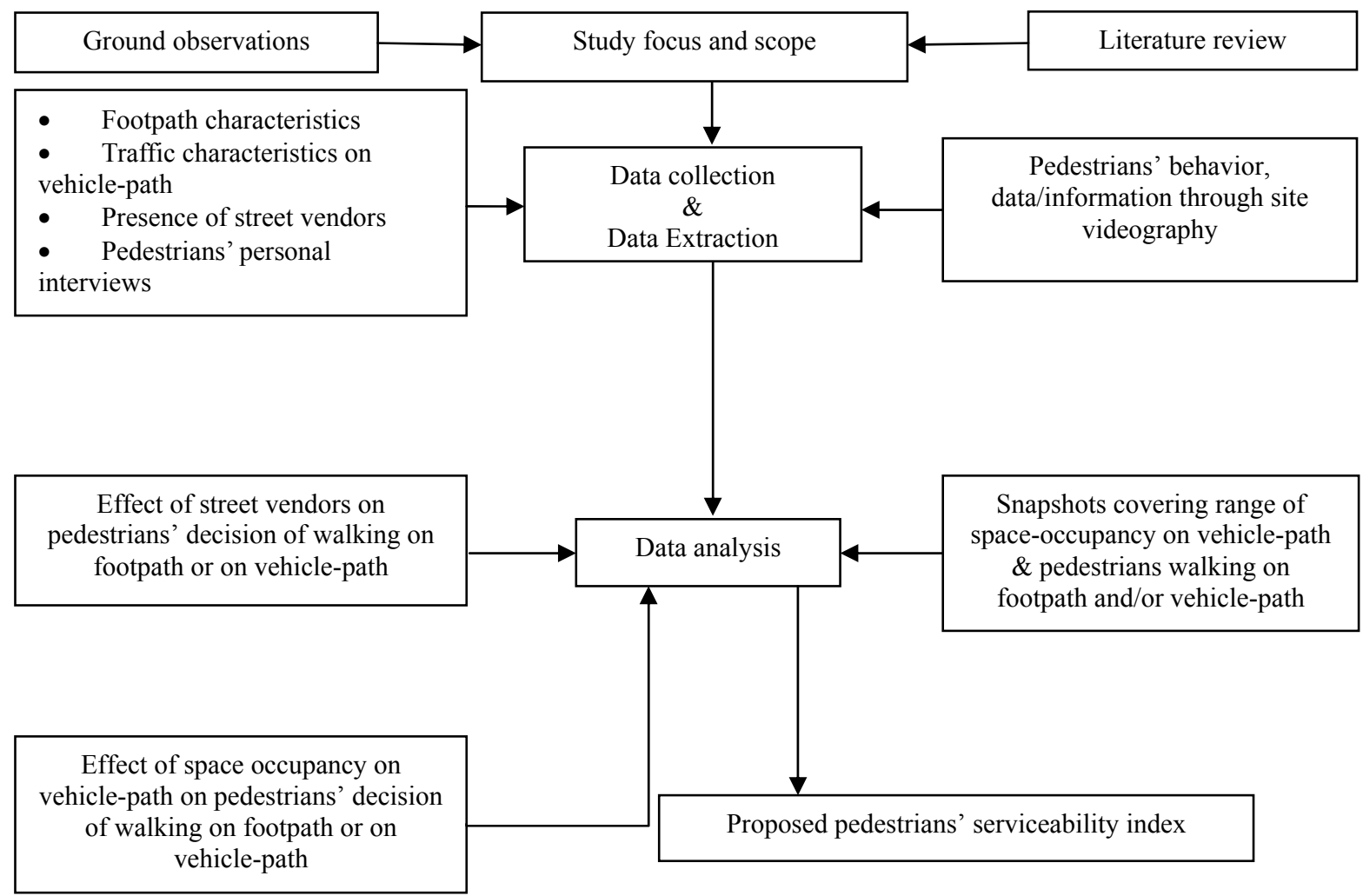

Fig. 1 Concept methodology.

\section{Data Collection and Extraction}

For this study, data have been collected at two locations in New Delhi, India. One of them is a two-lane undivided road having raised footpath on both the sides (Location 1, a segment of National Highway 2 near Badarpur border) with the motorized path width of 5.6 m (Fig. 2).

As shown in Fig. 2, width of footpath running in the direction from Okhla towards Faridabad (left half) is $1.8 \mathrm{~m}$ and width of footpath running in the direction from Faridabad towards Okhla (right half) is $1.2 \mathrm{~m}$. Here it is to be mentioned that by name it is a national highway but functionally it is a typical urban road. The footpath running in the direction from Okhla to Faridabad is of poor quality both in terms of physical characteristics (presence of surface irregularities, discontinuities) and environment (unpleasant, smelly, and congested by heavy pedestrian traffic). Also $1.5 \mathrm{~m}$ width of the vehicle-path adjoining the $1.8 \mathrm{~m}$ wide footpath, running in the direction from Okhla to
Faridabad is occupied by street vendors (vendors selling fruits, eatables, other articles on hand carts), who are observed in the site after morning peak hour.

The second location is a four-lane divided road with raised footpath on both the sides (Location 2, a typical arterial road, i.e., C.V.Raman Marg, Maharani bagh, New Delhi). Footpath width on both the sides is $1.8 \mathrm{~m}$. The line sketch is presented in Fig. 3.

Video-graphic data collected at study locations with trap length of $10 \mathrm{~m}$ (trap length is the distance between two imaginary lines considered for all observations of traffic speed, flow, density, etc.) for 2 hours of peak time (8:30 a.m. to 10:30 a.m.) and 2 hours of off-peak time (2:00 p.m. to 4:00 p.m.), captures classified traffic volume on vehicle-path, number of pedestrians walking on each footpath and vehicle-path and details of location and space occupied by street vendors. Apart from these, pedestrians' opinion survey has been carried out at same locations but on different days.

From the recorded videotapes at the two lane undivided road; 428 snapshots/stills have been 


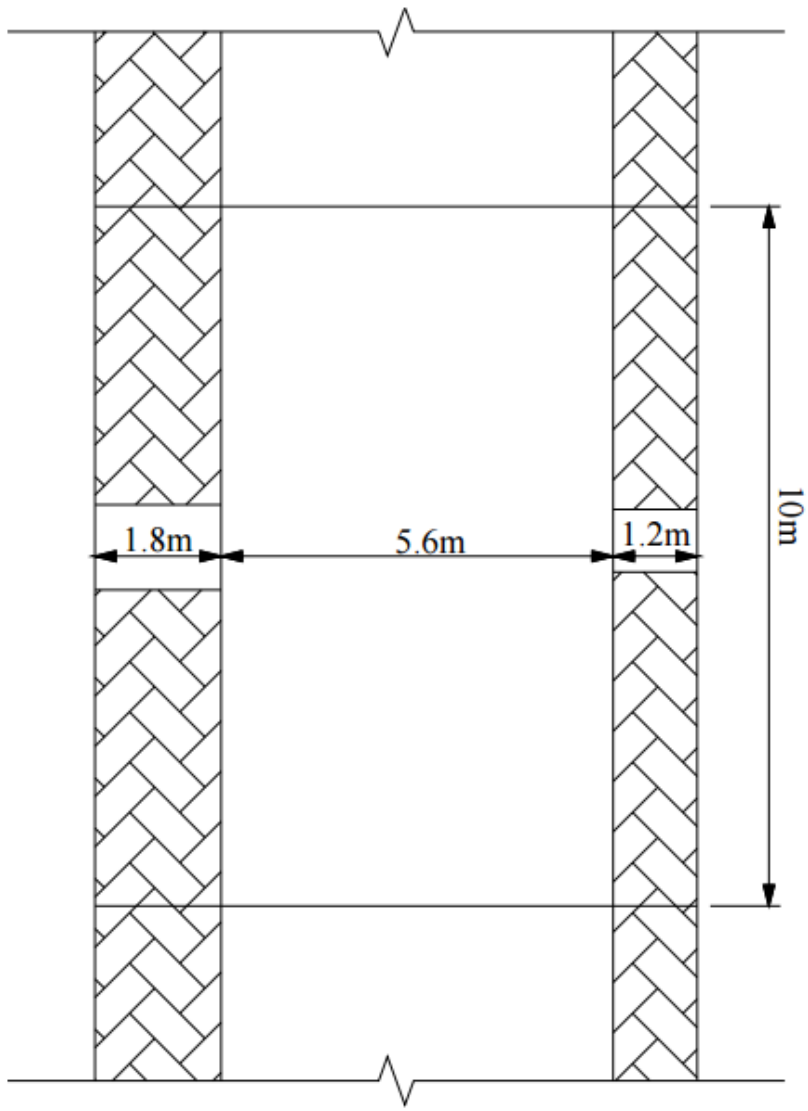

Fig. 2 Line sketch of location 1, i.e., two-lane undivided road.

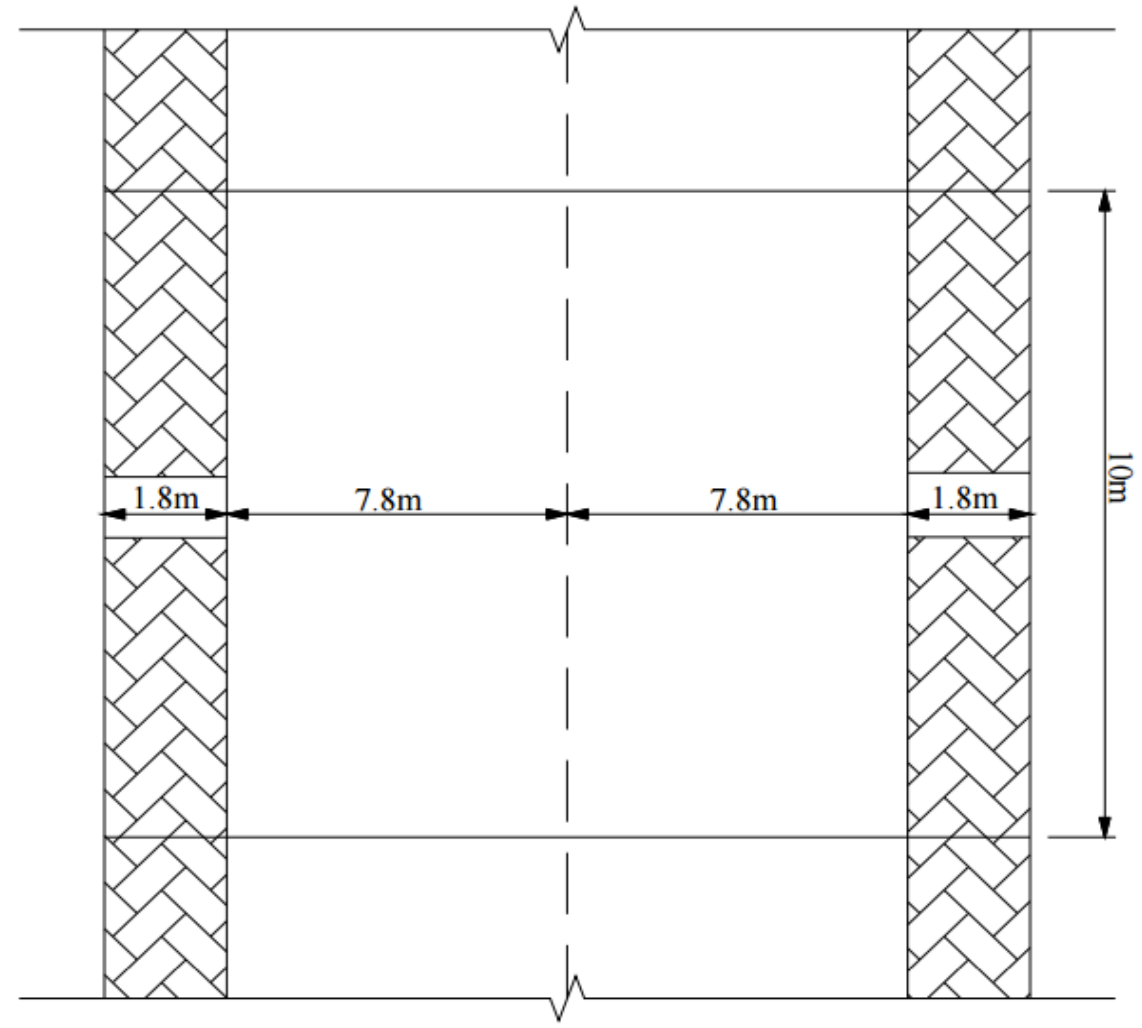

Fig. 3 Line sketch of location 2, i.e., four-lane divided road. 
captured randomly to include the widest possible range of space occupied by vehicles on vehicle-path based on observer's experience and judgment. Pedestrian flow of a minute time for which snapshot has been captured has been extracted from the videotape, for example, if snapshot taken is of timestamp 09:12:15.32 hours, then pedestrians' flow for a minute time, i.e., from 09:11:00 hours to 09:12:00 hours has been used. Further, number of pedestrians walking on left footpath, right footpath, left half of the vehicle-path and right half of the vehicle-path have been counted separately. Similarly, 371 snapshots have been captured for Location 2, i.e., with four lane undivided road.

\section{Traffic Characteristics at Study Locations}

Table 1 presents classified traffic volume count at both the locations. Data at Location 1 with two-lane undivided road is based on bidirectional traffic volume counts as it is an undivided road and data pertaining to Location 2 shows the vehicle counts for each direction as well as sum of both the directional counts. Road at the location with four-lane undivided road is much wider than road with two-lane undivided road and accordingly vehicle counts at location with four-lane undivided road is higher in absolute numbers. However, number of pedestrians at location with two-lane undivided road is higher.

\section{Space Occupancy on Vehicle-Path and Pedestrians' Walking Behavior}

At both the locations, $10 \mathrm{~m}$ trap length and width of road forms a rectangle, which has been used for calculation of percentage of space occupied by vehicles on vehicle-path. For each of 428 snapshots of Location 1 and 371 snapshots of Location 2, number and type of vehicles within this rectangle has been noted and accordingly percentage space occupied by vehicles and street vendors at both the locations during different points of time has been calculated based on standard vehicle sizes. Also, number of pedestrians walking on footpath as well as on vehicle-path is noted for all snapshots. Since Location 1 is an undivided road, it is divided by an imaginary line along the longitudinal direction into two halves. The $1.8 \mathrm{~m}$ wide footpath and

Table 1 Classified traffic volume counts at Location 1 and Location 2.

\begin{tabular}{lllll}
\hline \multirow{2}{*}{ Vehicle type } & \multicolumn{3}{c}{ Location 1 (bi-directional) } & \multicolumn{2}{c}{ Location 2 (direction wise and total) } \\
\cline { 2 - 5 } & $\begin{array}{l}\text { Morning peak hours } \\
(8: 30 \text { a.m. to 10:30 a.m.) }\end{array}$ & $\begin{array}{l}\text { Afternoon off-peak hours } \\
(2: 00 \text { p.m. to 4:00 p.m.) }\end{array}$ & a.m. to 10:30 a.m.) & $\begin{array}{l}\text { Afternoon off-peak hours } \\
(2: 00 \text { p.m. to 4:00 p.m.) }\end{array}$ \\
\hline Car & 188 & 190 & $1,202+855=2,057$ & $1,343+1,182=2,525$ \\
MTW (motorised two & 2,284 & 1,367 & $1,079+665=1,744$ & $1,367+1,183=2,550$ \\
wheeler) & 456 & 421 & $164+128=292$ & $1,288+1,598=2,886$ \\
Cycle rickshaw & 366 & 140 & $171+186=357$ & $976+1,078=2,054$ \\
Cycles & 95 & 130 & $122+126=248$ & $117+107=224$ \\
Auto rickshaw & 12 & 30 & & 574 \\
Bus/trucks & 64 & 107 & 1,114 & \\
Other vehicles & 5,964 & 3,867 & & \\
Pedestrians & & & & \\
\hline
\end{tabular}

Table 2 Observed footpath quality at Locations 1 and 2.

\begin{tabular}{ll}
\hline Location & Footpath quality observed visually \\
\hline Location 1 (right half) & Very good \\
Location 1 (left half) & Very poor \\
Location 2 (left half) & Poor \\
Location 2 (right half) & Good \\
\hline
\end{tabular}




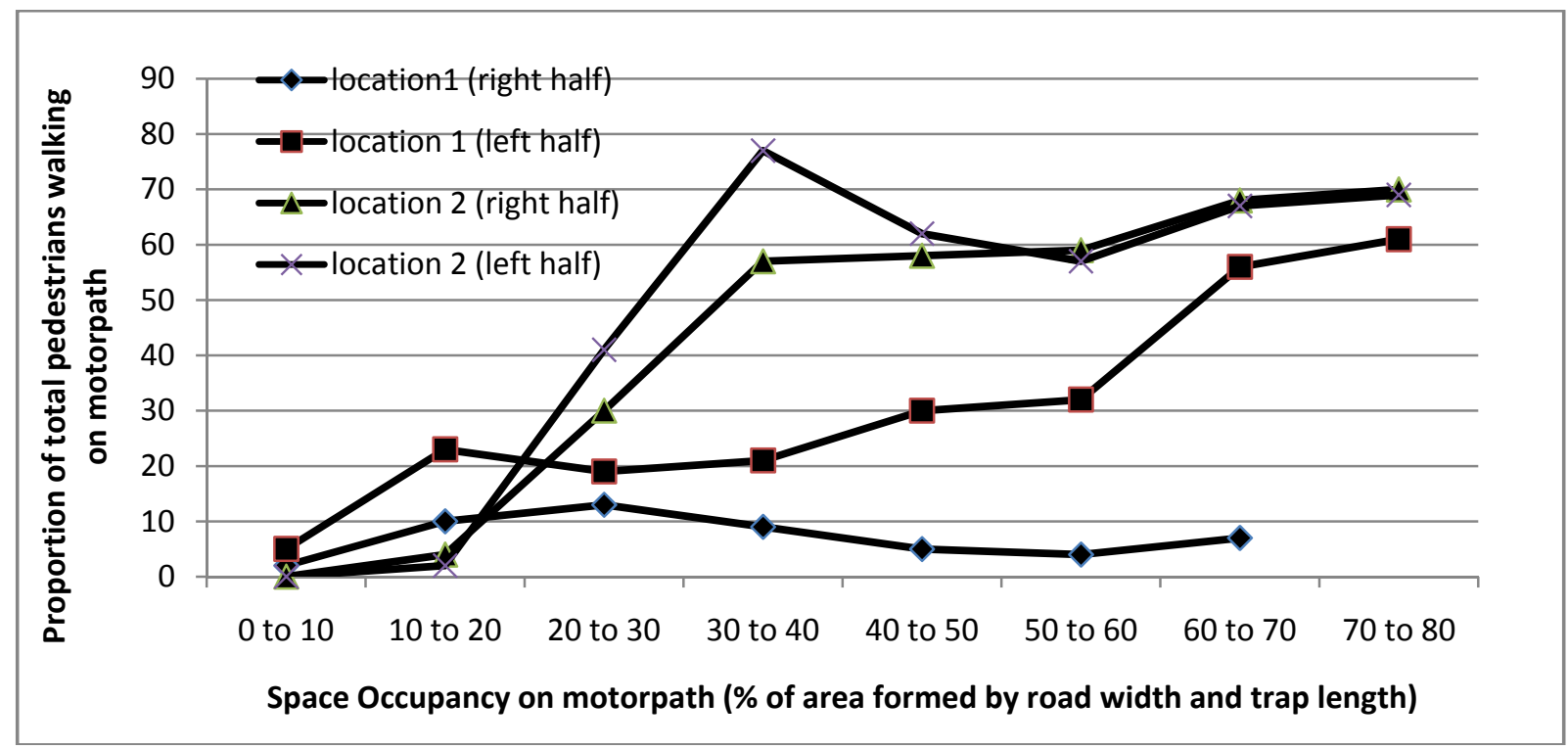

Fig. 4 Pedestrians' choice of walking on footpath with respect to space occupancy on vehicle-path.

$2.7 \mathrm{~m}$ width of vehicle-path adjacent to the footpath depicted as left half and the remaining $2.7 \mathrm{~m}$ width of vehicle-path and $1.2 \mathrm{~m}$ wide footpath is depicted as right half. Each half represents different road traffic environment. The right half being the area, where pedestrians are observed walking on the footpath only and no vendors are observed, whereas left half is the area where pedestrians are observed walking on the footpath as well as on the vehicle-path and vendors are observed occupying space on the vehicle-path after morning peak hours.

Accordingly, separate analysis for each half of the road at Location 1 has been carried out, using the captured 428 snapshots. At Location 2, two footpaths have been analyzed separately as it is a divided road. Further, visually observed footpath quality at all four footpaths have been noted as below in Table 2 .

For Locations 1 and 2, left and right half of the roads have been analyzed separately and Fig. 4 shows the space occupied by vehicles and/or street vendors on vehicle-path vs. the percentage of total pedestrians walking on vehicle-path.

It can be seen from Fig. 4, at Location 2; for medium space occupancy on vehicle path, i.e., $20 \%$ to $50 \%$; approximately $60 \%$ to $70 \%$ pedestrians are walking on vehicle-path. This indicates that walking on vehicle-path is preferable choice of pedestrians during medium space occupancy at vehicle-path as during low and high space occupancy pedestrians prefer not to walk on vehicle-path [19].

At Location 1 for right half, very less proportion of pedestrians are walking on vehicle-path irrespective of space occupancy on vehicle-path. This may be due to the fact that footpath quality is very good at this site. However, for left half, proportion of pedestrians walking on vehicle-path is not only high but it increases with increased space occupancy on vehicle-path. Here, it is to be noted that increased space occupancy on vehicle-path is due to the presence of street vendors. One of the reasons for more pedestrians walking on vehicle-path can be the presence of street vendors as pedestrians are observed purchasing from street vendors especially during off-peak hours. Accordingly, left half of Location 1 has been further analyzed separately for morning peak and afternoon off peak hours as street vendors are observed only during afternoon off peak hours.

The interrelationship among vehicular traffic, street vendors and pedestrian traffic shows that, more pedestrians (percentage) are walking on vehicle-path in the afternoon off-peak hours (2:30 p.m. to 4:30 p.m.) in the presence of street vendors as compared to the 


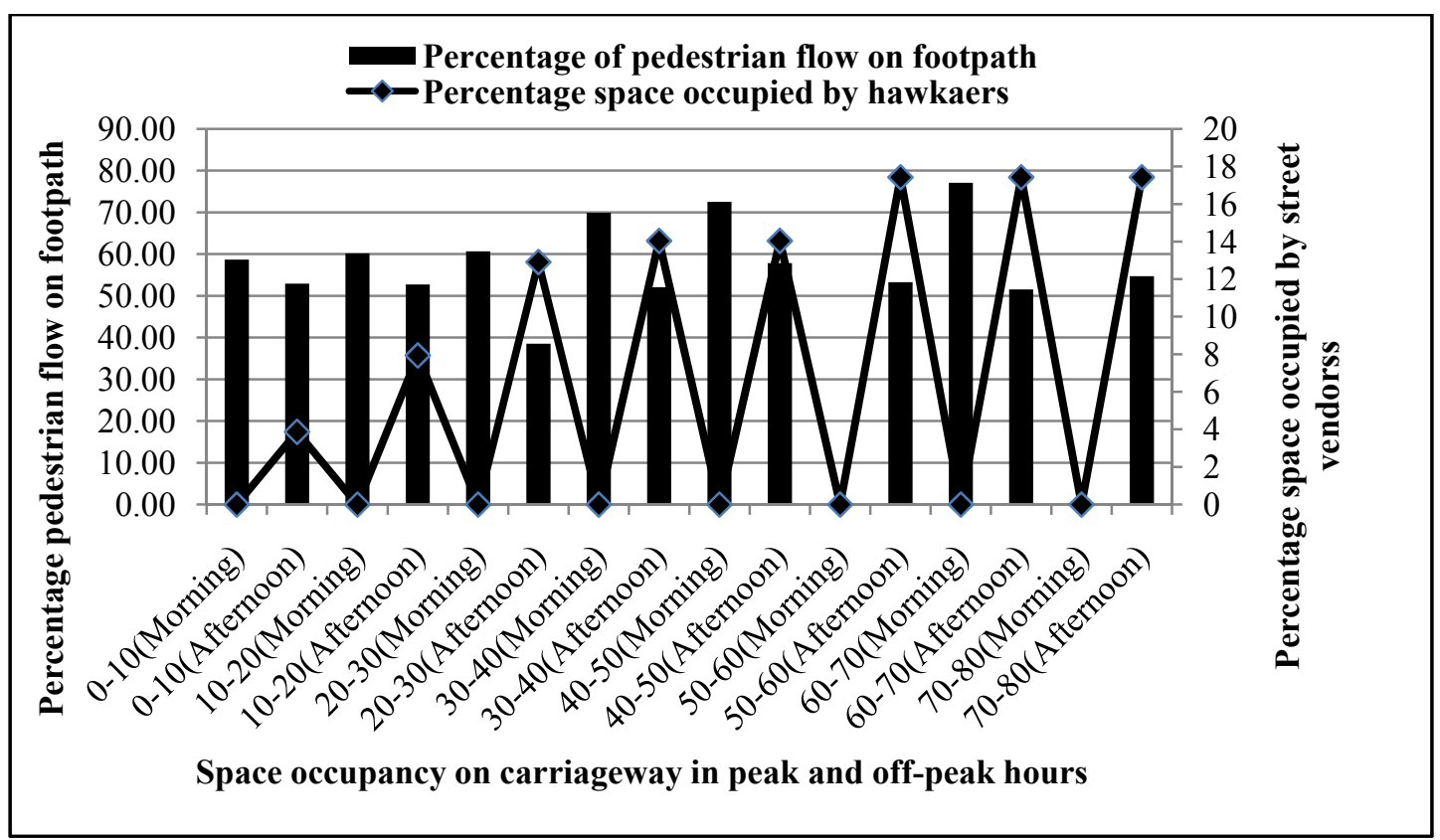

Fig. 5 Interaction among pedestrian flow, space occupied by vehicles and space occupied by street vendors during peak and off-peak hours on left half portion of the vehicle-path (Location 1).

morning peak hours (8:30 a.m. to 10:30 a.m.), for the same range of vehicle space occupancy (Fig. 5).

\section{Pedestrians' Opinion Survey}

Pedestrians' opinions have been collected through on-site personal interviews. Pedestrians were asked for the reason of their walking on footpath or vehicle-path. As expected, pedestrians have given multiple reasons for their choice.

At Location 1, a sample of 250 pedestrians has been taken for opinion survey. This covers pedestrians walking on left/right footpath as well as vehicle-path. Of the pedestrians using the left half portion of the road (i.e., left footpath and left half of the vehicle path), $72 \%$ belong to the age group of 18 to 40 years, $68 \%$ are male and $70 \%$ are making a work related trip. Of the pedestrians walking on left half of the vehicle-path, $63 \%$ are of the opinion that, "footpath surface is not good" and $35 \%$ are stating that width of footpath is not sufficient for which they have chosen the vehicle-path to walk on. $55 \%$ of them have stated that due to the presence of vendors on vehicle-path, they have chosen it to walk. Only $12 \%$ of the pedestrians walking on footpath are stating that the footpath on left half is comfortable to walk on and $61 \%$ of them are saying that they are walking on the footpath to avoid the heavy/risky traffic on vehicle-path. It is to be noted here that the majority of pedestrians walking on vehicle-path are walking there because of two reasons, i.e., poor footpath quality and presence of street vendors on vehicle-path. At the same time, pedestrians walking on footpath are walking there primarily due to unfavorable traffic conditions on vehicle-path. At this location, no pedestrians were observed walking on the right half of vehicle-path during the period of opinion survey and also the users of the footpath on right half were found satisfied.

At Location 2, 194 pedestrians were interviewed. This sample includes pedestrians walking on footpaths of left/right side and vehicle-paths of left/right side. At Location 2, no street vendors have been observed on vehicle-path. Out of 194, 74 were observed walking on vehicle-path and 120 on footpath. Out of 74 walking on vehicle-path, 59 mentioned "bad quality of footpath" as the reason for not walking on footpath. Response received from 120 pedestrians 
walking on footpath was primarily divided in two groups, i.e., 89 of them were walking on footpath because unfavorable traffic conditions on vehicle-path (either congested or unsafe). Remaining 31 gave different reasons for walking on footpath including "we should walk on footpath", "footpath is safer from vehicles", etc.

\section{Correlation among Parameters}

Except at Location1 (left half), all other locations have been observed where space on vehicle-path has been occupied by vehicles only. At Location 1 (left half), apart from vehicles, space has been occupied by street vendors as well during off-peak hours. Accordingly, effect of street vendors' presence on pedestrians' decision of choosing footpath or vehicle-path is analyzed by developing correlation matrix shown in Table 3 for the data observed at Location 1 (left side) separately. Correlation among space occupied by vehicles, street vendors, total pedestrian flow and percentage share of pedestrians walking on footpath has been studied and presented through correlation matrix (Table 3 ).

Results show the significant correlation between percentage of pedestrians walking on footpath and percentage space occupied by street vendors as also observed in the descriptive analysis. The negative sign $(-0.276)$ shows the inverse relationship, i.e., with increase in the percentage of space occupied by street vendors, less pedestrians are walking on footpath. This indicates that while making walk trip on this stretch, pedestrians are willing to use various facilities provided by street vendors. Since, percentage space occupied by street vendors shows significant correlation with all remaining three variables, regression is carried out by considering only one variable, i.e., space occupied by street vendors. Effect of percentage space occupied by street vendors, with respect to low or high percentage of pedestrians walking on footpath, has been checked with binary logistic regression. The dichotomous variable created is "low/high". The instances of percentage share of pedestrians walking on footpath less than $50 \%$, are categorized as "low" and if it is more than $50 \%$, it has been categorized as "high". With $p$-value of 0.001 , it is evident that influence of street vendors in pedestrians' decision making behavior regarding walking on footpath or on vehicle-path is significant (Table 4).

Table 3 Correlation among variables at Location 1 (lefthalf) where street vendors are present during off-peak hours.

\begin{tabular}{|c|c|c|c|c|c|}
\hline & & PER_Footpath & PER_space_veh & Per_space_street vendors & Total_flow \\
\hline \multirow{3}{*}{ PER_footpath } & Pearson correlation & 1 & -0.054 & $-0.276^{* *}$ & $0.252^{* *}$ \\
\hline & Sig. (2-tailed) & & 0.562 & 0.003 & 0.006 \\
\hline & $\mathrm{N}$ & 117 & 117 & 117 & 116 \\
\hline \multirow{3}{*}{ PER_space_veh } & Pearson correlation & -0.054 & 1 & $0.769^{* *}$ & $-0.272^{* *}$ \\
\hline & Sig. (2-tailed) & 0.562 & & 0.000 & 0.003 \\
\hline & $\mathrm{N}$ & 117 & 117 & 117 & 116 \\
\hline \multirow{3}{*}{$\begin{array}{l}\text { Per_space_stree } \\
\text { t vendors }\end{array}$} & Pearson correlation & $-0.276^{* *}$ & $0.769^{* *}$ & 1 & $-0.458^{* *}$ \\
\hline & Sig. (2-tailed) & 0.003 & 0.000 & & 0.000 \\
\hline & $\mathrm{N}$ & 117 & 117 & 117 & 116 \\
\hline \multirow{3}{*}{ Total_flow } & Pearson correlation & $0.252^{* *}$ & $-0.272^{* *}$ & $-0.458^{* *}$ & 1 \\
\hline & Sig. (2-tailed) & 0.006 & 0.003 & 0.000 & \\
\hline & $\mathrm{N}$ & 116 & 116 & 116 & 116 \\
\hline
\end{tabular}

** Correlation is significant at the 0.01 level (2-tailed);

PER_footpath = percentage of pedestrians walking on footpath $(\%)$;

PER_space_veh = percentage of space occupied by vehicles $(\%)$;

Per_space_street vendors $=$ percentage space occupied by street vendorss $(\%)$;

Total_flow $=$ total pedestrian flow $(\mathrm{ped} / \mathrm{min})$. 
Table 4 Results of binary logistic regression.

\begin{tabular}{llllllll}
\hline & & B & S.E. & Wald & df & Sig. & Exp(B) \\
\hline \multirow{2}{*}{ Step 1 } & Per_space_street vendors & 0.087 & .027 & 10.142 & 1 & .001 & 1.090 \\
& Constant & -1.537 & .288 & 28.509 & 1 & .000 & .215 \\
\hline
\end{tabular}

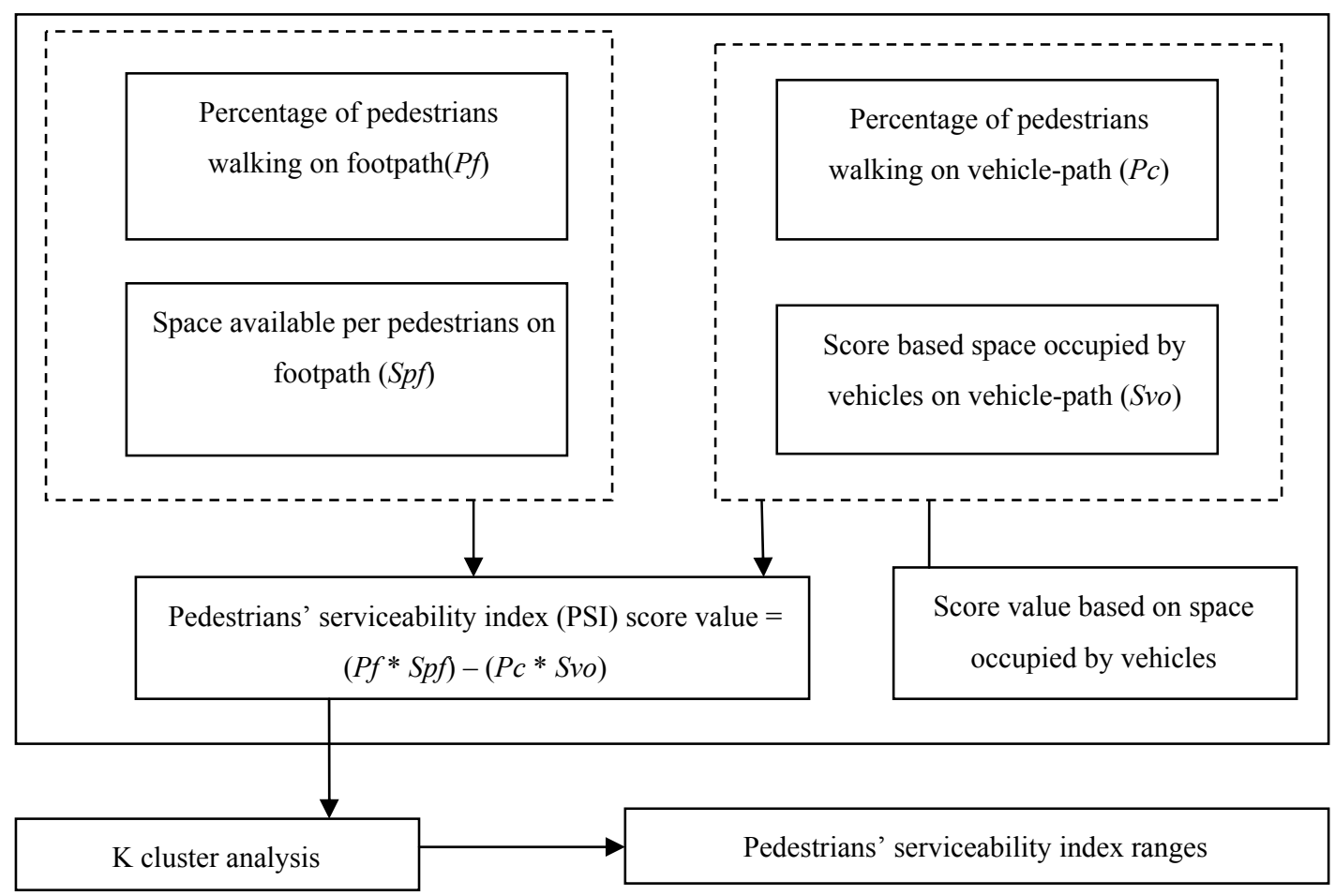

Fig. 6 Methodology chart for pedestrian level of service model formulation.

From Table 4, it can be interpreted that for approximately $9 \%$ of space occupied by street vendors, the majority (more than 50\%) of pedestrians are walking on vehicle-path.

\section{Proposed Method for Pedestrains' Serviceability Index Including Pedestrians Walking on Footpath as Well as on Vehicle-Path}

As discussed in previous sections, apart from footpath characteristics, vehicle characteristics, i.e., space occupancy on vehicle-path is playing a role in pedestrians' decision regarding walking on footpath or on vehicle-path. Low space occupancy crating high speed becomes unsafe and high space occupancy leads to congestion and unsuitable for walking. Based on all these parameters, pedestrians make decision of walking on footpath/vehicle-path. However, this does not get reflected in conventional methodologies of measuring pedestrians' level of service. This study proposes a method for measuring serviceability available to pedestrians, i.e., PSI (pedestrian serviceability index) on roads including walking on footpath and/or vehicle-path. The methodology chart for the proposed pedestrians' serviceability index is presented in Fig. 6.

As presented in methodology, proposed PSI includes percentage of pedestrians walking on footpath and also a factor for percentage of pedestrians walking on vehicle-path (Eq. (1)):

$$
\begin{gathered}
\text { PSI score }= \\
{\left[\left(P f^{*} S p f\right)\right]-\left[\left(P c^{*} S v o\right)\right]}
\end{gathered}
$$

where,

Pf $=$ percentage of pedestrians walking on the footpath;

$S p f=$ space available per pedestrian on footpath; 
$P c=$ accounts for percentage of pedestrians walking on vehicle-path (It is 0 if no pedestrians are walking on the vehicle-path and 1, if pedestrians are observed on the vehicle-path);

$S v o=$ score for space occupied by vehicles (on vehicle-path).

The factor, "percentage of pedestrians walking on footpath, $P f$ ", is a representative of pedestrians' preference of walking on footpath. If percentage of pedestrians walking on the footpath is more, it indicates that pedestrians are satisfied from comfort and safety aspects. "Space available per pedestrian" indicates the ability of the footpath to accommodate pedestrians and also the ability to provide ease of maneuvering to the pedestrians. Ease of maneuvering is directly related to speed of walking. Hence, the combined effect of these factors, "percentage of pedestrians walking on footpath" and "Space available per pedestrian" depicts the suitability of the footpath environment to the pedestrians. Space occupancy on vehicle-path affects pedestrians' decision making behavior regarding walking on footpath or on vehicle-path. Therefore, the combined effect of the factors "percentage of pedestrians walking on vehicle-path" and "space occupied by vehicles on vehicle-path" depict the suitability of vehicle-path for pedestrians.

$P f$ and $P c$ are taken from the extracted data. $S p f$ is calculated based on the pedestrian density and effective width of footpath. According to Parida [20], when space available per pedestrian on footpath is more than
$5.45 \mathrm{~m}^{2}$ then the facility is providing LOS A. Hence all the values of "Spf" considered for calculation of PSI is subjected to a maximum of $5.45 \mathrm{~m}^{2}$ in the present study.

At both the locations, it is observed that medium space occupancy levels at vehicle-path are comfortable for pedestrians to walk on vehicle-path compared to low and high space occupancy levels. At low vehicle space occupancy speed of vehicles is high, which is unsafe for pedestrians. At high vehicle space occupancy, speed of vehicles is less, traffic comes near to stand -still position, which becomes uncomfortable for pedestrians to walk on and find their way. At medium vehicle space occupancy, speed of vehicles is moderate and it is not unsafe and uncomfortable for pedestrians to walk on the vehicle-path; still there are some associated risks. In the present study, the range of $20 \%-50 \%$ is taken as the medium vehicle space occupancy range. As the space occupied by vehicles increases or decreases from this range, it creates discomfort and safety concerns respectively for the pedestrians and therefore those ranges of vehicle space occupancy have been assigned with progressively higher values. This representation of pedestrians comfort should be included in measuring their level of service. Table 5 presents a relative score of pedestrian comfort for the different range of space occupancy levels, i.e., space occupancy at vehicle-path. The maximum value of "Svo" considered is 65 and the minimum is 35 .

Table 5 Score for vehicle space occupancy (Svo).

\begin{tabular}{ll}
\hline Vehicle space occupancy on vehicle-path & Score for space occupancy $($ Svo $)$ \\
\hline Less than $10 \%$ & 65 \\
$10 \%-20 \%$ & 55 \\
$20 \%-30 \%$ & \\
$30 \%-40 \%$ & 35 \\
$40 \%-50 \%$ & \\
$50 \%-60 \%$ & 55 \\
$60 \%-70 \%$ & 65 \\
$70 \%-80 \%$ & 65 \\
$80 \%-90 \%$ & 65 \\
More than $90 \%$ & 65 \\
\hline
\end{tabular}


The minimum value of $P S I$ as per Eq. (1) would be "-65" when no pedestrian is there on the footpath and the maximum value of $P S I$ score would be " 545 " when all pedestrians are walking on the footpath. Freezing the maximum and minimum values of PSI score, a data series for expected PSI score has been developed. Grading operation is carried out on the developed series using K-cluster analysis, to define 6 ranges for 6 levels of service as shown in Table 6 . The standard deviation and mean of each of the 6 clusters are determined. The upper limit of PSI score for each level is determined by adding twice the standard deviation to the respective cluster centers and the lower limit is determined by subtracting 1.5 times standard deviation from the respective cluster centers. The proposed criterion for PSI measurement is developed accordingly.

\section{Applying Proposed PSI to Locations 1 and 2}

Developed model has been applied to two locations considered in this study to assess its $P S I$ as well as PSI score if footpath quality has been improved to its best and all pedestrians' walks on footpath only. In order to check the PSI that the pedestrian walking environment is providing, 117 and 98 snapshots are chosen based on observers' experience and judgment for Locations 1 and 2, respectively, such that each data point belongs to a different vehicle space occupancy ranging from 0 to value close to $100 \%$ and corresponding percentage of pedestrians walking on the footpath. The PSI for the existing road traffic scenario of the road environment is checked with Eq. (1) by calculating PSI score for each snapshot location-wise. The PSI of the assumed

Table 6 Pedestrians' serviceability index ranges.

\begin{tabular}{ll}
\hline PSI levels & PSI score range \\
\hline$P S I$ "A" & $\geq 374.40$ \\
$P S I$ "B" & $215.2-374.40$ \\
$P S I$ "C" & $95.95-215.2$ \\
$P S I$ "D" & $19.10-95.95$ \\
$P S I$ "E" & $(-) 23.64-19.10$ \\
$P S I$ "F" & $\leq(-) 23.64$ \\
\hline
\end{tabular}

Table 7 Comparison of both the scenarios at Location 1 (left half).

\begin{tabular}{llll}
\hline$P S I$ & $P S I$ score & $\begin{array}{l}\text { Number of snapshots in each PSI in existing } \\
\text { scenario }\end{array}$ & $\begin{array}{l}\text { Number of snapshots in each } P S I \text { when } 100 \% \\
\text { pedestrians are on footpath }\end{array}$ \\
\hline PSI A & $\geq 374.40$ & 5 & 42 \\
$P S I$ B & $215.20-374.40$ & 40 & 58 \\
$P S I C$ & $95.95-215.2$ & 57 & 17 \\
$P S I$ D & $19.10-95.95$ & 14 & 0 \\
$P S I$ E & $(-) 23.64-19.10$ & 1 & 0 \\
$P S I F$ & $\leq(-) 23.64$ & 0 & 0 \\
\hline
\end{tabular}

Table 8 Comparison of both the scenarios in terms of PSI at Location 2.

\begin{tabular}{llll}
\hline$P S I$ & $P S I$ score & $\begin{array}{l}\text { Number of snapshots in each PSI in existing } \\
\text { scenario }\end{array}$ & $\begin{array}{l}\text { Number of snapshots in each PSI when 100\% } \\
\text { pedestrians are on footpath }\end{array}$ \\
\hline$P S I$ A & $\geq 374.40$ & 5 & 42 \\
$P S I$ B & $215.20-374.40$ & 40 & 58 \\
$P S I$ C & $95.95-215.2$ & 57 & 17 \\
$P S I$ D & $19.10-95.95$ & 14 & 0 \\
$P S I$ E & $(-) 23.64-19.10$ & 1 & 0 \\
$P S I \mathrm{~F}$ & $\leq(-) 23.64$ & 0 & 0 \\
\hline
\end{tabular}


scenario, i.e., all pedestrians are walking on the footpath after improving the footpath quality is determined by adding the number of pedestrians walking on vehicle-path to the number of pedestrians walking on footpath for each of the snapshots and calculating PSI score for each. Both the scenarios are compared and presented in Table 7.

Similarly, Table 8 shows results for Location 2.

From Tables 7 and 8, it can be inferred that the PSI of the pedestrian facility is improving when pedestrians are being confined to the footpath itself. It would improve further if the width of footpath is increased and vending zone is installed as street furniture.

\section{Conclusion}

This study develops PSI to assess pedestrians' walking including pedestrians walking on footpaths as well as on vehicle-path of the same road. The study identifies factors affecting pedestrians' decision of walking on footpath or on vehicle-path which include footpath quality, traffic characteristics (Space occupancy) on vehicle-path and presence of street vendors. It also brings the range of space occupancy on vehicle-path where pedestrians have chosen to walk on footpath compared to walking on vehicle-path. Developed PSI is based on these parameters and applicable to roads where pedestrians may/may not be walking on footpath.

\section{Acknowledgments}

The authors of the paper are grateful to Director, CSIR - Central Road Research Institute and Champion of the project entitled "Development and Application of Technologies for Sustainable Transportation (SUSTRANS)" for granting permission to publish this paper. Also the authors are thankful to Council of Scientific and Industrial Research (CSIR) for sponsoring the study SUSTRANS (ESC-0106). All results are sub-set of the above ongoing research study.

\section{References}

[1] Asadi-Shekari, Z., Moeinaddini, M., and Shah, M. Z. 2013. "Non-motorized Level of Service: Addressing Challenges in Pedestrian and Bicycle Level of Service." Transport Reviews 33 (2)..

[2] Fruin, J. J. 1971. "Designing for Pedestrians: A Level-of-Service Concept." Highway Research Record: Journal of the Highway Research Board 355: 1-15.

[3] National Research Council, Transportation Research Board. 2000. Highway Capacity Manual. Washington, D.C.

[4] National Research Council, Transportation Research Board. 2010. Highway Capacity Manual. Washington, D.C.

[5] Lautso, K., and Murole, P. 1974. "A Study of Pedestrian Traffic in Helsinki: Methods and Results." Traffic Engineering and Control 1974: 446-9.

[6] Sarkar, S. 1993. "Determination of Service Levels for Pedestrians, with European Examples." Transportation Research Record: Journal of the Transportation Research Board 140: 35-42.

[7] Henson, C. 2000. "Level of Service for Pedestrians." ITE Journal 70 (9): 26-30.

[8] Gallin, N. 2001. "Quantifying Pedestrian Friendliness - Guidelines for Assessing Pedestrian Level of Service." Presented at Walking the 21st Century, 20th to 22nd February, 2001. Perth, Western Australia.

[9] Mozer, D. 1994. "Calculating Multi-modal Levels-of-Service.” Accessed December 8, 2017. http://www.ibike.org/engineering/los.htm.

[10] State of Florida Department of Transportation. 2009. Quality/Level of Service Handbook. Accessed December 10, 2017. https://www.hayward-ca.gov/sites/default/files/ documents/Appendix_HCM2000_FDOT_Capacity_Table s.pdf.

[11] Landis, B. W., Vattikuti, V. R., Ottenberg, R. M., McLeod, D. S., and Guttenplan, M. 2001. "Modeling the Roadside Walking Environment: A Pedestrian Level of Service." Transportation Research Record: Journal of the Transportation Research Board 173: 82-8.

[12] Underlien, J. S. 2007. "Pedestrian and Bicycle Level of Service on Roadway Segments." Accessed December 8, 2017. http://seattlegreenways.org/wp-content/uploads/Pe destrian-and-Bicyclist-Level-of-Service-on-Roadway-Seg ments.pdf.

[13] Jaskiewicz, F. 2000. "Pedestrian Level of Service Based on Trip Quality." TRB Circular E-C019: Urban Street Symposium: Number E-C019.

[14] Abley, S., and Turner, S. 2011. Predicting Walkability. 
NZ Transport Agency research report.

[15] Muraleetharan, T., Adachi, T., Uchida, K., Hagiwara, T., and Kagaya, S. 2004. "A Study on Evaluation of Pedestrian Level of Service along Sidewalks and at Crosswalks Using Conjoint Analysis." Journal of Infrastructure Planning 21 (3): 727-35.

[16] Petritsch, T. A., Landis, B. W., McLeod, P. S., Huang, H. F., Challa, S., Skaggs, C. L., Guttenplan, M., and Vattikuti, V. 2006. "Pedestrian Level-of-Service Model for Urban Arterial Facilities with Sidewalks." Transportation Research Record: Journal of the Transportation Research Board 1982: 84-9

[17] Dowling, R., Flannery, A., Landis, B., Petritsch, T., Rouphail, N., and Ryus, P. 2008. "Multimodal Level of Service Analysis for Urban Streets." Transportation
Research Record: Journal of the Transportation Board 2071: 1-7.

[18] Patnaik, S., Advani, M., and Parida, P. 2015. "Why and When Pedestrians Walk on Carriageway in the Presence of Footpath?-A Behavioral Analysis in Mixed Traffic Scenario of India." Presented at 8th Urban Mobility India Conference and Expo, November 24th 27th, 2015, New Delhi, India.

[19] Advani, M., and Nisha, G. 2013. "Behavioral Analysis of Pedestrians for Walking on Footpath and on Vehicle-Path in 'Space-Sharing' Traffic Scenario." Indian Highways: Journal of the Indian Road Congress, 41-7.

[20] Parida, M. 2015. Review Report for Indian Highway Capacity Manual. CSIR-Central Road Research Institute, New Delhi, India, August 2nd, 2015. 